$\xi_{p-1}$

\title{
Automated Elephant Entry Prevention for Human and Crop Protection
}

\author{
Mahalakshmi K ${ }^{1}$, Kodanda Ramaiah G ${ }^{2}$, Nandha Kishore ${ }^{3}$, K. Ramesh ${ }^{4}$ \\ ${ }^{1,2 \& 3}$ Department of Electronics and Communication Engineering -Kuppam Engineering College, Kuppam, Andhra, India \\ ${ }^{4}$ Department of Electrical \& Electronics Engineering, Kuppam Engineering College, Kuppam, Andhra, India \\ *Corresponding author E-mail: mahakailash123@gmail.com
}

\begin{abstract}
Farming in areas with elephants has been the norm in many parts of Asia for thousands of years. In particular, farmers have settled closer to areas where elephants are present and started growing crops. Elephant raids occur in the night time where people do not notice the presence of an elephant until it starts an off ensive behavior. Such raids damage the crops, harvest storages and homes in addition to causing deaths of humans. A multitude of traditional methods have been developed through the ages to reduce and prevent crop raiding by elephants like shouting, drum-beating, noise-making, use of fire crackers, electric fencing, lights and torches. Such activities may also indicate to elephants that their presence is detected, and that they have to contend with aggressive humans. In the proposed system, automated detection of the presence of elephant is done by analyzing the infrasonic sounds produced by elephants and they are prevented from entering into farms by propagating the offensive sounds in low frequencies without human intervention.
\end{abstract}

Keywords: Crop protection, elephant entry prevention, infrasonic sound, rumble, pre-processing, feature extraction.

\section{Introduction}

Farming in areas with elephants has been the custom in many parts of Asia for thousands of years. Cultivated crops represent varieties of plants that have been artificially selected and raised to increase their nutritional value, productivity, and to decrease plant secondary compounds. As such, crops are much more attractive to herbivores than wild fodder. Wherever crops are cultivated in areas with elephants, crop raiding is an overriding factor [Fig. 1] and farmers from time immemorial have devised ways and means of guarding their crops from raiders. Rural villagers store their crop harvests inside their houses, which attracts elephants into those domestic areas in dry seasons. $98.3 \%$ of such elephant raids occur in the night time where people do not notice the presence of an elephant until it starts an offensive behavior. Such raids damage the crops, harvest storages and homes in addition to causing deaths of elephants and humans. Residents of the areas affected by elephant raids have resorted to legal as well as illegal counter measures. Such legal solutions range from implementing electric fences covering domestic areas to using re crackers to drive away elephants. Electric fences are often subjected to breakages and maintaining such fences requires considerable financial and human effort. Therefore, rural villagers also practice various illegal and destructive methods to protect their property such as placing poisoned vegetables and shooting crop raiding elephants. Few wild species suggest as much attention and varied emotions from humans as elephants. Their imposing size, high level of intelligence and complex social behavior attracts attention and endearment while their propensity to raid crops and sometimes aggressive behavior also instils fear and animosity. The Asian elephant (Elephas Maximus) is a symbol of pride, status, and cultural heritage throughout its geographical range. Historically the elephant has been revered and worshipped as a God, used as a warrior, ambassador, beast of burden, and has held a central place in Asian civilizations. Today it is one of the leading 'flagship species for biodiversity conservation in Asia.

However, friction between humans and elephants termed HumanElephant Conflict (HEC) occurring mainly over space and manifesting in seasonal raids in villages that lie in the elephants' range often result in retaliatory killings. This has come to seriously threaten their survival in the wild. If left unchecked, it will result in the demise of an icon of Asia's heritage. Elephants and human were built with a long tradition of interdependence up until contemporary times. However, today Human-Elephant Conflict (HEC) has become one of the most significant socio-ecological problems in some parts of the world. The conflict primarily is a consequence of frequent attacks from crop riding elephants on the rural agricultural communities. The consequences of HEC made loss of human lives or permanent disability, damage to the properties and cultivation while making negative attitude towards the elephants. Subsequently, elephant deaths resulted from avenging killing by people has become ever increasing number during past few years, making Asian elephants (Elephas Maximus) categorized as Endangered in the International Union for Conservation of Nature (IUCN). For example, Farmers in Andhra, Chittoor district, are now apprehensive of herds of wild elephants raiding their fields. In recent times, the frequency of adventurism by elephants in the fields has increased to at least once a week. Farmers are spending sleepless nights as they lay awake, in case herds of wild elephants enter the fields and destroy crops ready to harvest. According to recent census, there are 22 elephants in Chittoor west division and 20 to 25 in the east division. Ramakuppam has jumbos from AP whereas Gudipalli Mandal has elephant's crossing over from Karnataka. Wild elephants come to Kuppam from Tamilnadu forest 
and V.kota has similar visitors belonging to the Pernambattu forest area. All these areas share borders with other states. The automated acoustic detection of elephants is an important factor in the prevention of wild animal's entry into the farms. The consequences of human-elephant conflict are not only of key conservation concern but also are major socio-economic and political issues. Therefore, resolution of human-elephant conflict is a major concern and a high priority for conservation of elephants in range countries.

Influx of humans and conversion of natural habitat to human dominated land-use causes fragmentation and loss of elephant habitat. With increased contact, elephants progressively raid crop fields and break down houses to get at stored crops. Chance encounters between elephants and people, as well as efforts of people to guard against elephant depredation results in injury and death of humans. Harmful methods employed by people in the

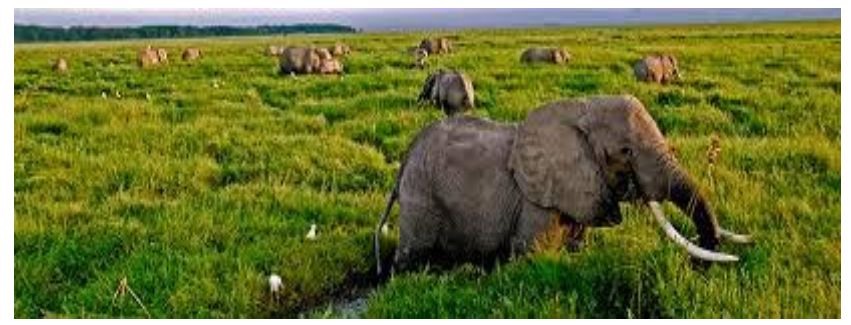

process result in death and injury of elephants thereby escalating human-elephant conflict.

Fig.1: Crop Raiding by Elephants

Today, the main issue with prevention of crop raiding by elephants is not whether or not it can be achieved, but rather how it can be actualized at a favorable cost-benefit ratio. In most cases, the problem is that the economic value of crops cultivated is very low. Although the total loss due to elephants can be considerable, the damage per-unit of cultivation is generally very low. Therefore, while many methods can be utilized to effectively safe guard crops from elephants the cost per unit of safeguarding can be comparatively high, preventing their employment on a wide scale. A multitude of traditional methods [Fig.2] have been developed through the ages to reduce and prevent crop raiding by elephants in conflict prone areas. The growth of human-elephant conflict in the past few decades and technological advances have resulted in development of additional methods to address the problem. In general, traditional methods are easy to use, have low costs and are more effective at low levels of conflict. With increasing conflict, more technical and sophisticated methods need to be used which carry higher costs. The various techniques employed in humanelephant conflict mitigation range from chasing elephants by shouting, drum-beating, noise-making, use of fire crackers, lights and torches, to engaging koomkies (trained elephants) and specially trained and equipped teams of people, construction of elephant barriers such as rubble walls, ditches and canals, biological and electric fences, deployment of alarms, development of communication systems, capture, translocation and culling of problem animals [2], use of highly sophisticated technology such as satellite telemetry, and compensation and insurance schemes.

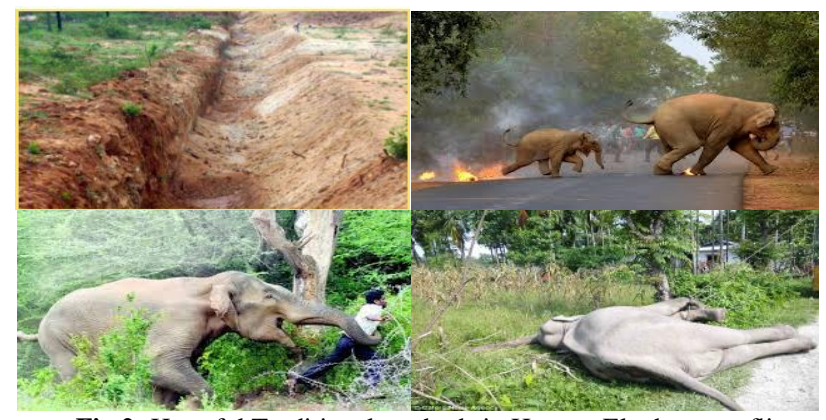

Fig.2: Harmful Traditional methods in Human-Elephant conflict
Activities such as noise-making, shouting and throwing objects are more reactive and confrontational. Such activities may also indicate to elephants that their presence is detected, and that they have to contend with aggressive humans. Lighting fires has been a universal method of guarding crops against elephants and other wild animals since ancient times Presence of humans, noise making, fires etc. keep elephants away from crop fields or their vicinity by presenting clues that are clearly associated with humans. Such activities tend to lose their effectiveness in protecting crops as elephants become habituated with increased exposure to them, and the realization that such methods are not backed by any real physical threat or harm. Males appear to habituate to traditional methods of crop protection more readily than females in herds. When these methods fail - as they are bound to with eventual habituation - the animosity towards elephants begin to increase, as does the perception of the crop-degradation being significant. Furthermore, confrontational methods increase the risk of injury and death to farmers from elephants as the aggression levels increase on both sides.

\section{Related Works}

No one method is a 'stand-alone' universal solution for conflict resolution/mitigation. Each technique has its advantages and disadvantages. Methods may be used in differing permutations to increase their effectiveness. Farming practices, traditions and expectations of people, environmental conditions, habitat characteristics, resource availability and even elephant behavior may vary widely across the range of Asian elephants. Thus local information is vital to determine what methods will be appropriate for a given situation. Being intelligent and highly adaptable animals elephants also learn to avoid and overcome many of the methods used for mitigation, and methods that were initially successful may lose their effectiveness over time. Therefore, continuous monitoring and adaptive management based on results is critical for successful human elephant conflict mitigation. Most activities conducted to safe guard crops and to mitigate the humanelephant conflict have been developed almost entirely from the point of view of human needs. The impact of such activities on elephants has been rarely studied. Some activities such as elephant translocation and range restriction with barriers, though usually conducted with the idea of safeguarding elephants, may be extremely detrimental to their survival when used inappropriately. Therefore, it is essential to not only monitor the success of activities in mitigating the conflict but also to study their direct and indirect impacts on the elephants concerned.

Today, however, elephants remain under threat from poaching (Douglas Hamilton 2008; Lemieux and Clarke 2009), habitat loss and the resulting human- elephant conflict, which refer to the problem that elephants destroy crops, damage houses and sometimes even kill people. Farmers, in return, react by shooting, wounding and killing elephants (Hoare and Toit 1999; Santiapillai et al.2010). The human-elephant conflict is a serious conservation problem in Africa and Asia. Due to the rising number of elephants and the increasing human population, the habitat of elephants becomes increasingly narrow.

Due to the lack of habitat, elephants enter new territory, which often coincides with agricultural areas or human villages. Different efforts have been undertaken to alleviate this conflict, such as the establishment of electric fences, which is, however, not practicable to cover larger areas. Early warning systems are required that monitor travel routes of elephants and alert humans to avoid involuntary confrontations. Elephants communicate with each other by low-frequency sounds, which travel distances of several kilometers'. The most common elephant call is the rumble, which extends into the infrasound band.

The rumble is a harmonic sound with a fundamental frequency in the range of $15-35 \mathrm{~Hz}$ and duration between 0.5 and 5s [15]. Fig. 3 shows a typical rumble with a high signal-to-noise ratio (SNR). 
The acoustic detection of elephants by their calls is currently the most promising approach towards an early warning system that is able to detect the presence of elephants over large distances. However, the large variety of noise sources present in the wild delay automated analysis methods. As a result, no system exists so far that is ready to operate in the field. So far research on acoustic analysis of elephant calls has addressed highly selective tasks, such as the identification of elephants by their calls and the analysis of particular call types, e.g. rumble types. The automated detection of elephant calls, which is the basis for the above mentioned tasks, has rarely been investigated. There are two major challenges in the detection of elephants in wildlife recordings. The first challenge is the large variety of uncontrollable noise sources. Noise originates for example, from wind, rain, cars, and airplanes, which particularly pollute the low-frequency channel where elephant calls reside. Additionally, human speech and sounds from other animals disturb the automated detection. The second challenge is the sparsity and irregularity of elephant calls, which makes it difficult to predict the occurrence of a call. The contribution of this is a robust method for the detection of elephant presence. For this purpose, an audio representation that models psycho acoustic properties of the elephant's hearing system is employed. A novel method is introduced for the enhancement of signal quality to improve the noise robustness of the representation. The detector is evaluated on a large dataset of wildlife recordings to simulate a real-life scenario.

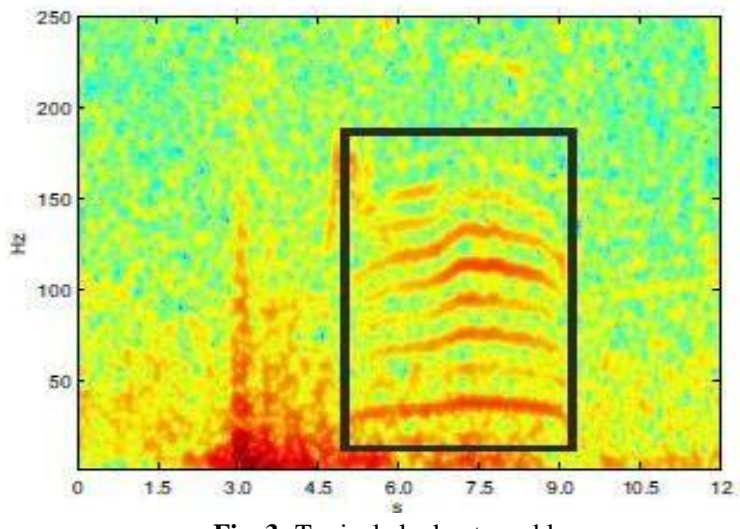

Fig. 3: Typical elephant rumble

A solution to the human-elephant conflict is not complete without a system to detect and localize elephants. The sounds emitted by elephants can be used to detect them. It has been three decades since the first discovery of infrasonic waves emitted from Asian Elephants [12]. Elephants emit various kinds of vocalizations such as rumbles and trumpets. Those vocalizations start from very low frequencies which are considered as infrasonic into audible sounds. In contrast to the other frequencies in elephant vocalizations, the importance of infrasonic emissions lies in the fact that lower frequencies of sounds travel longer distances than their audible counterparts. Therefore, infrasonic emissions from wild elephants can open a new door for detecting and locating them over a significant distance, which has many benefits. For example, in order to build electric fences, it is necessary to clearly identify the movement patterns of elephants closer to domestic areas that need to be protected. Improper laying of electric fences makes them ineffective in protecting humans and waste deployment costs. Furthermore, long distance detection and localization can help to warn villagers about raids from aggressive elephants where electric fences are not available or not functioning properly. In this paper the preliminary results of effort in detecting and localizing elephants using the infra-sounds emitted by them is presented.

To achieve this goal, there is need to record infra-sound, ensure that it is really emitted by an elephant and then finally use a localization method to locate the elephant. This work contributes to a larger system that we are devising to address some causes of the humanelephant conflict. Elephants require relatively large areas and a diversity of environments to forage (van Aarde et al. 2008; Santiapillai et al. 2010). Therefore, their ranges are complex and not confined to officially designate protected areas. In Kenya, elephants have distinct home sectors linked by travel corridors that typically cross unprotected areas (Douglas-Hamilton et al. 2005). In Southern Africa, landscape fragmentation forces elephants into clustered conservation areas across several countries (van Aarde et al. 2007), which leads to local overpopulation and perceived adverse consequences for vegetation (Pienaar et al.1966; Hanks 1979).

Progressive conservation approaches aim at providing corridors between core areas to facilitate elephant's natural movements. Such corridors require (Taylor \& Francis) autonomous systems that detect elephants area-wide and continuously monitor their migration patterns. In addition, elephants approaching human settlements need to be detected in real-time so that actions can be made in a timely manner. Elephants make extensive use of powerful low-frequency vocalizations commonly termed 'rumbles' (Poole et al. 1988; Langbauer 2000; Soltis 2010), which travel distances of up to several kilometres (Garstang 2004). This qualifies the elephant as a perfect model species for acoustic observation as it is possible to detect elephants by their rumbles even if they are out of sight (Payne et al. 2003; Seneviratne et al. 2004). The automated analysis of animal vocalizations has recently received increasing research attention as a method to study and monitor wild animals without interfering with their lives or habitat (Blumstein et al. 2011). Thompson, Schwager and Payne (2009) showed that the calling rate of low-frequency elephant vocalizations is a useful index of elephant numbers, demonstrating that acoustic surveying is a valuable tool for estimating elephant abundance, as well as for detecting other vocal species and anthropogenic noises that may be associated with poaching. The accuracy of elephant localization and detection significantly depends on the characteristics of sound propagation in the air. The sound propagates through the air as longitudinal waves and the propagation is affected by the properties of the medium.

For instance, the variations in the metrological parameters such as temperature, wind, relative humidity etc. results in the sound propagation variations in terms of intensity and speed. Dissanayake C.M et al. [4] analyzed major propagation constraints that could affect the elephant localization framework. The SNR has much more considerable effect on the source localization accuracy in our algorithm. The sound intensity depletes with the distance, resulting lower SNR levels at the sensors when the source is far away. The system provides enormously inaccurate position estimations once the SNR level drops under certain marginal level. More precisely, the level is $-12 \mathrm{~dB}$ with respect to simulation framework. Therefore, in actual implementation, incorporating signal processing techniques for noise reduction will increase the overall performance of the system.

Payne et al.[12] discovered that most of the fundamental frequencies of acoustic calls from elephants are in the range between $14 \mathrm{~Hz}$ to $24 \mathrm{~Hz}$. They point out that the higher frequencies, which Asian elephants can hear the best (about $1000 \mathrm{~Hz})$, attenuate more significantly than the lower frequencies(less than $30 \mathrm{~Hz}$ ) in the presence of trees and thick grass. Payne et al. also note that Asian elephants hear infrasonic calls better than high frequencies if the distance from the source is greater than $300 \mathrm{~m}$. Langbauer et al.[9] have shown this by playing back low frequency calls of elephants from distance about $2 \mathrm{~km}$ and observing the responses of the elephants to these infrasonic emissions. This shows the importance of using infrasonic calls to detect elephants from longer distances.

Fundamental frequencies of elephant infrasonic calls generate higher frequency harmonics, resulting a wide spectrum of audible sounds. Zeeppelzauer et al.[20] present a signal enhancement technique for identifying sounds from elephants in the presence of various types of noise sources such as wind and automotive engines. They have used a large dataset that consist of prior recordings in such noisy environments with the presence of 
elephants. According to their observations, higher frequency harmonics of the fundamental infrasonic vocalizations decrease when the distance from elephants to the infrasonic microphone increases. The observation agrees with the conclusions by Payne [12] as these experiments were conducted in an environment with similar conditions.

Sound detection has a long history. There are two general approaches to sound detection: template-based methods and feature-based methods. Template based methods successively match a given sound example (template) to a (longer) sound recording, in order to find occurrences of the template in the recording. A straight-forward approach is the matched filter method where two spectrograms are directly matched to each other. The method is optimal to find occurrences of the template itself in the recording, but suboptimal if similar signals to the template should be found or complex noise sources are present[17]. D. K. Mellinger et al. [10] propose the spectrogram correlation technique, which employs more abstract templates to make the matched filter more robust. The templates represent the coarse spectro-temporal energy distribution of the searched-for sound and improve the tolerance of the matching process.

The spectrogram correlation technique has been applied to elephant call detection in [17]. However, results are reported to be suboptimal. One reason is that elephant calls vary significantly in duration and spectrogram correlation is not able to model variances in duration. Figure shows the large variation in duration of rumbles(from $0.5 \mathrm{~s}$ to $2.5 \mathrm{~s}$ ). A more promising template based method for call detection has been recently introduced in [7]. The authors perform semi-supervised learning to select the sound snippet (template) that best discriminates between the positive and negative sound samples in a provided training set. For sound detection, the spectrograms of the template and of the recording are compared to each other using a distance measure that builds upon MPEG compression [2] and which allows for a certain tolerance in time and frequency. The approach has not been applied to elephant calls so far. We evaluate the approach on elephant call detection and compare it with the proposed approach.

The second class of approaches for sound detection are featurebased techniques. The advantage of feature-based techniques over template-based techniques is the additional layer of abstraction introduced by the features, which provide a higher-level representation of the sounds. For the detection of elephant calls, feature-based techniques have been developed that build upon certain acoustic characteristics of elephant's rumbles. P.J.Venter et al. [17] exploit the harmonic structure of rumbles and perform pitch detection using a sub-band pitch analysis. The authors report good performance as long as the harmonic structure of the rumbles is not buried in background noise at least three harmonics can be clearly distinguished.

In practice, we observe that the harmonic structure of rumbles is often covered by noise, which is introduced by wind and other low-frequency disturbers like cars and airplanes. P.J.Venter et al. [17] report that engine noises lead to false positive detections if they have stronger harmonics than the rumbles. Fig 2.1 (a) shows a rumble in the presence of narrow-band noise introduced by a car engine. The engine sound has a harmonic at $70 \mathrm{~Hz}$, which is particularly misleading for detectors that rely on harmonic structures is hardly visible. The harmonic structure for short rumbles [Fig. 4(a) and Fig. 4(c)] is less salient than for rumbles with a longer duration.

Additionally, the number of harmonics decreases with the distance of the caller to the microphone. Fig. 4(b) \& 4(d) shows the noise and two distant rumbles where the higher harmonics are completely missing, which impedes pitch detection as reported by [17].

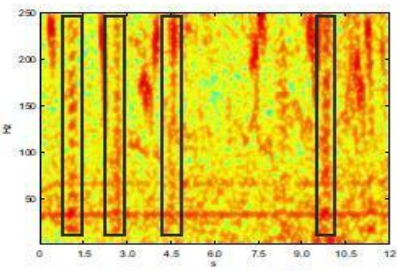

(a) harmonic noise

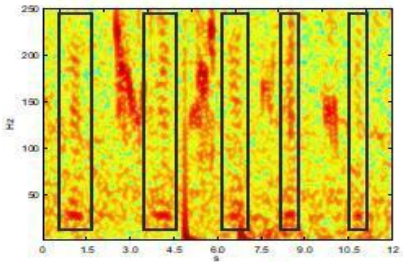

(c) short rumbles

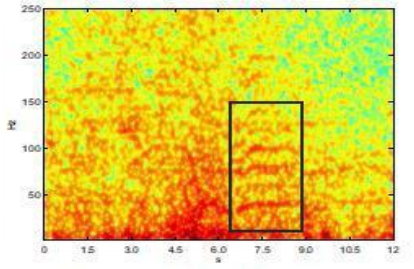

(b) broadband noise

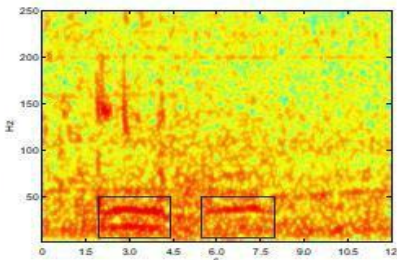

(d) missing harmonics
Fig. 4: Rumbles with different interfering noise sources

Based on these observations, J.Wijayakulasooriya [18] proposes formant analysis for the detection of elephant rumbles. The formants are derived from the peaks of transfer function of the all pole filter obtained by Linear Predictive Coding (LPC). The basic assumption of the approach is that the first and second formant tracks of a rumble, which is superimposed by narrow-band harmonic noise. The resulting formant tracks show a high variation over time of approximately $20 \mathrm{~Hz}$. The example in Fig. 5 shows a clean rumble with a strong temporal modulation.
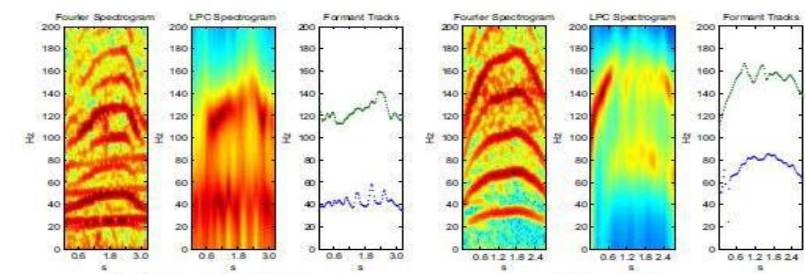

Fig. 5: Formant tracks of elephant rumbles

The formant tracks reflect this modulation, which results in a variation $30 \mathrm{~Hz}$. From the existing investigations we conclude that a more holistic representation of the frequency distribution required that does not rely on specific (and partially difficult to detect) sound attributes such as pitch, harmonics and formants.

A promising signal representation based on MFCC features has been proposed in [3]. The authors of [3] extend MFCCs by replacing the Mel-scaled filter bank with a more general Greenwood-scaled filter bank [6]. The Greenwood scale is a logarithmic scale that models the critical bands and that can be adapted to all mammals for which the corresponding hearing range is known (which is the case for elephants). Zeppelzauer et al. [20] investigate the detection of elephant calls in a real-life scenario. In this scenario a broad range of noise sources exist that decrease the signal-to-noise ratio. Additionally, the investigated calls originate from near and far distant elephants and from many different individuals. This results in a more complex setting for automated analysis than in [3].

Wijayakulasooriya.J.Z [18] proposed a method for the automatic detection of elephants by using a Hidden Markov Model (HMM). The infrasound calls have been used to automatically detect the presence of elephants. Commonly, the time- frequency distribution of the audio signal is used to detect the infrasound elephant call signatures. Fig. 6 (a) shows the waveform of an elephant infrasound call and the corresponding time-frequency distribution using Short Time Fourier Transform (STFT). The comb like pattern formed by parallel dominant frequency tracks shown in Fig. 6 (b) is unique to elephant calls and commonly used for detection of elephants. However, a number of field tests revealed that the frequencies in the range of $0-100 \mathrm{~Hz}$ gets highly 
contaminated with environmental noise, particularly the wind. Therefore, alternative techniques had to be investigated in order to obtain more efficient set of features for distinguishing elephant calls from other environmental noise.

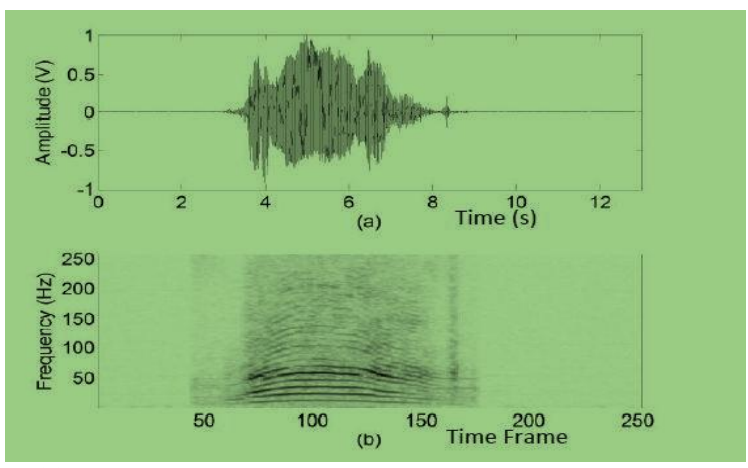

Fig. 6 (a): Audio waveform of an elephant infrasound call (b) Parallel dominant frequency tracks

\section{Proposed System}

A robust method for automated detection of elephant rumbles is proposed for the detection of specific sound attributes made by elephants. The detector is trained for rumbles directly from a small number of sound samples. The proposed detector yields high performance on wildlife and steps towards an automated detection system for elephant presence. In the proposed approach the input signal is framed and transformed into frequency domain. Next, signal enhancement is done by applying analysis to reduce the influence of noise. Then the extraction of formants of elephant calls from the enhanced signals, where the elephant calls was identified by feature extraction for accurate automatic recognition. The block diagram of the proposed method is shown in Fig. 7.

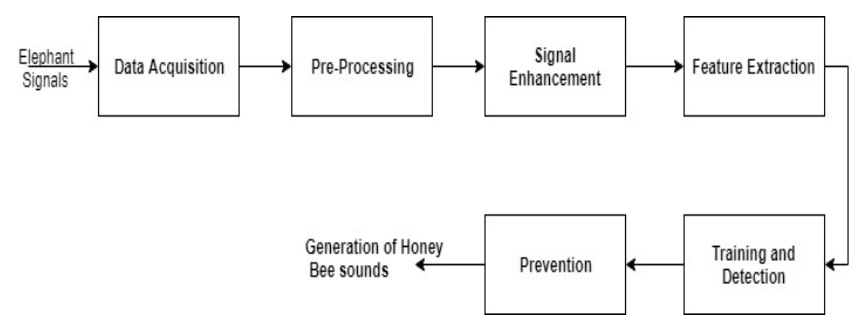

Fig.7: Block diagram for the Elephant Entry Prevention method

\section{a) Data Acquisition}

The signal captured by the microphone is well-suited as input to the elephant detector because it enables the detection of elephant calls coming from all direction. The elephant calls are generated as a result of the vibration of the vocal cords and then the sound travelling through the cavity of the elephant skull and trunk. Formants are the resonant frequencies of the vocal track and they can be used to recognize the presence of elephant calls like rumble, trumpet and snots.

The rumble is a harmonic sound produced by an elephant with in a fundamental frequency range of $15-35 \mathrm{~Hz}$ and a strongly varying duration (from $0.5 \mathrm{~s}$ to more than $10 \mathrm{~s}$ in our data). Depending on the distance of the caller, rumbles exhibit a varying number of harmonics. The fundamental frequency at approximately $20 \mathrm{~Hz}$ and numerous higher harmonics are clearly recognizable. From the capturing of wildlife recordings, it is observed that many noise sources corrupt the frequency band where rumbles reside. As a result, the fundamental frequency and the harmonic structure are masked to a high degree of noise sources like wind and rain, as well as engine sounds of cars and airplanes. The engine sound has a fundamental frequency at $30 \mathrm{~Hz}$, which is particularly misleading for detectors that rely on pitch detection. Rumbles are superimposed by broadband noise where the harmonic structure is hardly visible. The harmonic structure for short rumbles is less salient than that for rumbles with a longer duration. Sound of higher frequency is limited in range by atmospheric attenuation. As a consequence the number of harmonics decreases with the distance of the caller to the microphone.

\section{b) Pre-Processing}

The input signal obtained is converted to short audio frames and transform each framed signal into the Fourier domain by FFT. Since the energy of elephant rumbles is mostly concentrated below $500 \mathrm{~Hz}$ we limit the analyzed frequency range to $0-500 \mathrm{~Hz}$. The analysis window is set to $300 \mathrm{~ms}$ to capture the infrasound components with adequate frequency resolution. Temporal smoothness is obtained by a small step size between successive frames of $30 \mathrm{~ms}$.

Generally, the pre-processing stage in speech recognition systems is used in order to increase the efficiency of subsequent feature extraction and classification stages and therefore to improve the overall recognition performance. Commonly the pre-processing includes the sampling step, a windowing and a de-noising step. At the end of the pre-processing the compressed and filtered speech frames are forwarded to the feature extraction stage.

Due to increasing mobile use it is noticeable that speech recognition systems need to be robust with respect to their acoustic environment. Together with the feature extraction stage, the motivation of the pre-processing is to generate a parametric representation of the speech signal that is very compact and still stores all the necessary information for automatic speech recognition. In order that a computer is able to process the speech signal, it first has to be digitized. Therefore the time-continuous speech signal is sampled and quantized. The result is a time- and value-discrete signal.

According to the Nyquist-Shannon sampling theorem a timecontinuous signal that is band limited to a certain finite frequency $f$ $\max$ needs to be sampled with a sampling frequency of at least $2 \mathrm{f}$ max. In this way it can be reconstructed by its time-discrete signal. Previous Studies shown that the sampling frequency in combination with the feature vector size has a direct effect on recognition accuracy. Since human speech has a relatively low bandwidth (mostly between $100 \mathrm{~Hz}$ and $8 \mathrm{KHz}$ ) a sampling frequency of $16 \mathrm{KHz}$ is sufficient for speech recognition tasks. For the purpose of having a value discrete signal the sampled values are quantized. This leads to a significant reduction of data.

Usually speech recognition systems encode the samples with 8 or 16 bits per sample depending on the available processing power. 8 bit per sample would mean $28=256$ quantization levels, 16 bit per sample provide $216=65536$ quantization levels. Concluding, if you have enough processing power, a higher bit resolution for the sampled values is preferable. Speech is a non-stationary time variant signal. A short but precise explanation of stationary and non-stationary signals as well as the non-stationary nature of speech is given here. In brief, a signal is considered to be stationary if its frequency or spectral components do not change over time.

We assume that human speech is built from a dictionary of phonemes, while for most of the phonemes the properties of speech remain invariant for a short period of time $(\sim 5-100 \mathrm{~ms})$. Thus we assume (and hope) the signal behaves stationary for those time frames. In order to obtain frames we multiply the speech signal with a windowing function. This windowing function weights the signal in the time domain and divides it into a sequence of partial signals. By doing so we gain time information of every partial signal keeping in mind that an important step of the pre-processing and feature extraction is a spectral analysis of each frame. In prevention of an elephant from entering into farms, detection of an elephant sound plays an important role. From the researches, it is clear that elephant sounds are infrasonic sounds. Infrasound is sound below the level of human hearing. The frequency of a sound is measured in Hertz $(\mathrm{Hz})$ and the infrasonic range is generally considered to be between 1 and $20 \mathrm{~Hz}$. Elephant calls may contain frequencies ranging from a low of $5 \mathrm{~Hz}$ to a high over $10000 \mathrm{~Hz}$. 
From this, it is difficult to train the detector for the elephant calls whether the calls are infrasonic sounds or greater than the range of infrasonic sounds. To train the detector for the elephant calls, analysis of elephant sounds is necessary. Analysis part consists three sections: Pre- processing, Estimating power spectral density and Peak analysis. The block diagram for the analysis of a signal is as shown below in Fig.8.

The pre-processing stage is used in order to increase the efficiency of subsequent feature extraction and classification stages and therefore to improve the overall recognition performance. Commonly the pre-processing includes windowing the signal and removing noise from the signal.
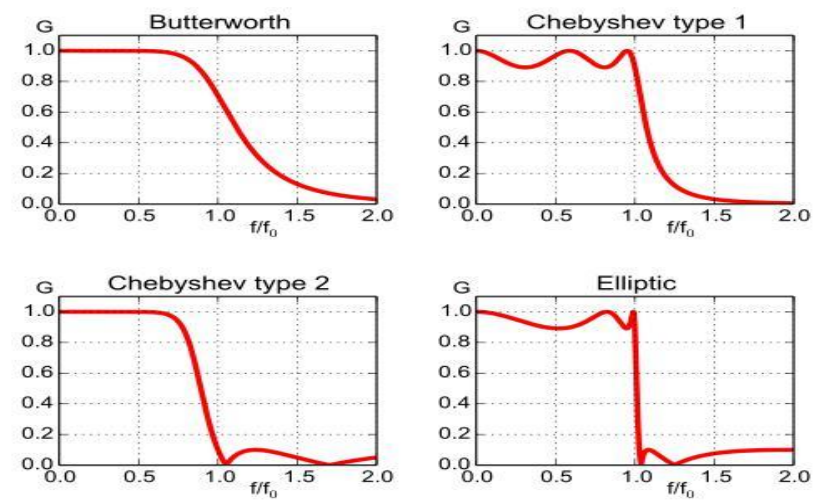

Fig.8: Block Diagram for Analysis of an Elephant Sound

i) Windowing the signal: In signal processing, a window function is a mathematical function that is zero- valued outside of some chosen interval. Window function for a signal describes the shape of its geometric representation. In both digital filter design and spectral estimation, the choice of a windowing function can play an important role in determining the quality of overall results. The main role of the window is to damp out the effects of the Gibbs phenomenon that results from truncation of an infinite series. Among all the filter functions in MATLAB, the filter using in this work is Elliptic filter. Elliptic filter is a signal processing filter with equalized ripple behavior in both pass band and stop band. The amount of ripple in each band is easily adjustable, and no other filter of equal order can have a faster transition in gain between the pass band and stop band.

ii) Elliptic filter: The elliptical filter has an extremely sharp cutoff frequency, which makes it ideally suited for filter design cases where there must be severe attenuation in frequencies just entering the stop-band of the filter. Further, because the rippling effect is distributed across both the pass- and stop-bands in the elliptic filter, it makes it an excellent candidate for a low pass filter where the amount of error needs to be minimized on both sides of the cutoff frequency. The Chebyshev filter, with a slower roll-off and an imbalanced ripple, does not offer either of these advantages. Therefore, in cases where there are signals that are very close and must be cut off at exactly or very close to one particular frequency, the elliptic filter is recommended.

The comparison of other filters with elliptic filters is as shown in Fig. 9.

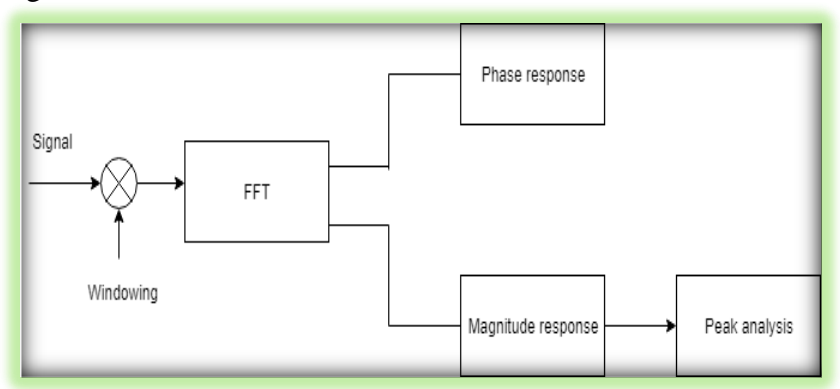

Fig. 9: Comparison of Elliptic Filter with Other Filters iii) Elliptic filter design in MATLAB: The function which is used to design elliptic filter is "ellip". The design of an elliptic filter is as follows

$[\mathrm{b}, \mathrm{a}]=\operatorname{ellip}(\mathrm{n}, \mathrm{Rp}, \mathrm{Rs}, \mathrm{Wp})$

Here, $\mathrm{n}$ - Order of a low pass elliptic filter

$\mathrm{Rp}-\mathrm{dB}$ of Pass band ripple

Rs $-\mathrm{dB}$ of Stop band ripple

Elliptic filter is used in this work to perform window function to the signal. Elliptic filter is a signal processing filter with equalized ripple behavior in both pass band and stop band. The amount of ripple in each band is easily adjustable, and no other filter of equal order can have a faster transition in gain between the pass band and stop band. The elliptical filter has an extremely sharp cutoff frequency, which makes it ideally suited for filter design cases where there must be severe attenuation in frequencies just entering the stop-band of the filter. Further, because the rippling effect is distributed across both the pass- and stop-bands in the elliptic filter, it makes it an excellent candidate for a low pass filter where the amount of error needs to be minimized on both sides of the cutoff frequency.

iv) Peak analysis: The signal obtained after noise reduction is a complete noise free signal. Fourier transform is applied to the noise free signal which gives the magnitude response and phase response. The magnitude response is considered to analyze the peaks in the signal which is termed as peak analysis. A Fast Fourier Transform (FFT) is an algorithm that samples a signal over a period of time and divides it into frequency components. These components are single sinusoidal oscillations at distinct frequencies each with their own amplitude and phase. An FFT computes the DFT and produces exactly the same result as evaluating the DFT definition directly; the most important difference is that an FFT is much faster.

\section{c) Signal Enhancement}

Environmental sounds, such as wind and rain generate broadband noise which reduces the signal-to-noise ratio. The background noise masks the fine harmonic structures of the rumbles and makes them hard to detect. In this work, noise from the signal is reduced with the help of digital filter.

From this work, signal to noise ratio of the signal can be increased. The stage of de-noising or noise reduction, also referred to as enhancing of speech degraded by noise, aims to improve the speech signals quality. The objective is to improve the intelligibility, a measure of how comprehensible speech is. Noise corrupting speech signals can be grouped coarsely into the following 3 classes:

1. Microphone related noise

2. Electrical noise (e.g. electromagnetically induced or radiated noise)

3. Environmental noise

The first two types of noise can be easily compensated by training the speech recognizers on corresponding noisy speech samples, but compensating the environmental noise is not that elementary, due to its high variability. The basic problem of noise reduction is to reduce the external noise without disturbing the unvoiced and low-intensity noise-like components of the speech signal itself.

\section{d) Feature Extraction}

In machine learning, pattern recognition and in image processing, feature extraction starts from an initial set of measured data and builds derived values (features) intended to be informative and non-redundant, facilitating the subsequent learning and generalization steps, and in some cases leading to better human interpretations. Feature extraction is related to dimensionality reduction. Feature extraction involves reducing the amount of resources required to describe a large set of data. When performing analysis of complex data one of the major problems 
stems from the number of variables involved. Analysis with a large number of variables generally requires a large amount of memory and computation power, also it may cause a classification algorithm to over fit to training samples and generalize poorly to new samples. Feature extraction is a general term for methods of constructing combinations of the variables to get around these problems while still describing the data with sufficient accuracy. Many machine learning practitioners believe that properly optimized feature extraction is the key to effective model construction.

\section{e) Training and detection}

The detector is trained on the aggregated features. The preferred classifier is a linear support vector machine (SVM). The linear SVM is chosen, since it provides good results even for small training sets, has only a few parameters to specify, and exhibits a strong generalization ability due to its low complex decision boundary. Additionally, the linear SVM has outperformed other classifiers (allowing more complex decision boundaries) in preliminary experiments.

\section{f) Prevention}

Once the elephant is detected, the system will be switched ON and the sounds like honeybee sounds and natural calamities sounds can be propagated through air and ground. Bees are known to be attracted to the water around elephant's eyes and when they get up their trunks, elephants can go berserk (out of control with anger or excitement).

\section{Results and Analysis}

Initially testing for the sinusoidal signals which are less than 30 $\mathrm{Hz}$ to analyse the low frequency signals is done. After analyzing the sinusoidal signals, the similar process is repeated for analyzing the elephant sound signals. Initially, the elephant sound signal is as shown in Fig. 10.

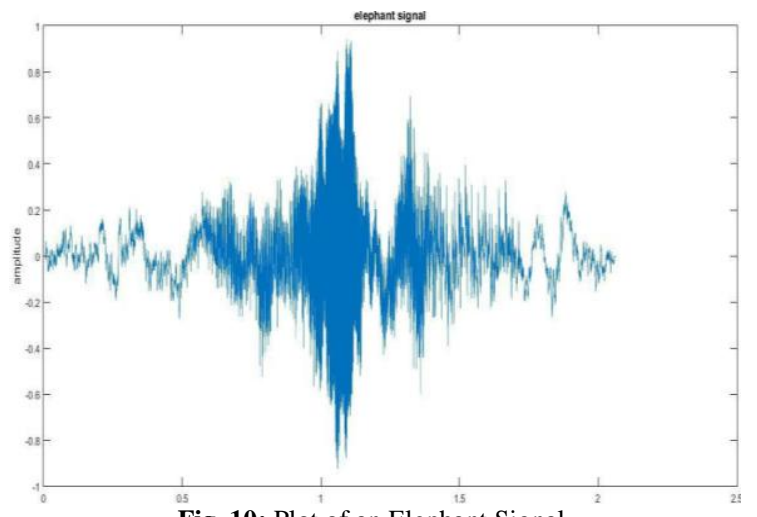

Fig. 10: Plot of an Elephant Signal

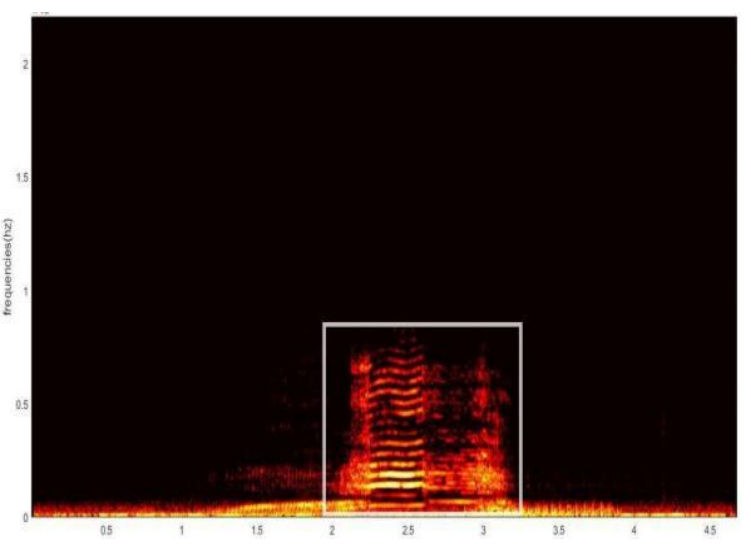

Fig.11: Spectrogram of an Elephant Signal
To analyze the signal, first the spectrogram is plotted for the above signal. The spectrogram is as shown in Fig. 11. From the above drawn spectrogram, it is clearly stated that, the frequencies of an elephant signal are of low values. The marked area in the above figure shows the area where the signal is having high power. But from the above figure, we could not determine the exact value of a frequency at where a peak is located. So we are going for the analysis of a signal by applying filter and FFT to the signal and finding peaks in the signal.

\section{a) Applying a filter}

Generally an elephant signal lie in the range of $5 \mathrm{~Hz}$ to a high of $470 \mathrm{~Hz}$. In most cases, the signal lies below $270 \mathrm{~Hz}$. So design a elliptic filter with a range of $300 \mathrm{~Hz}$. An elliptic filter is a signal processing filter with equalized ripple behavior in both the pass band and stop band. The amount of ripple in each band is independently adjustable, and no other filter of equal order can have a faster transition in gain between the pass band and stop band, for the given values of ripple. After applying elliptic filter to the elephant signal, the result is as shown in Fig. 12 (a) \& (b).

From the above figure, it is clearly visible that first signal is a sample signal of elephant sound and the second figure shows the output signal after applying an elliptic filter. Here filtering is applied to the signal to $300 \mathrm{~Hz}$.
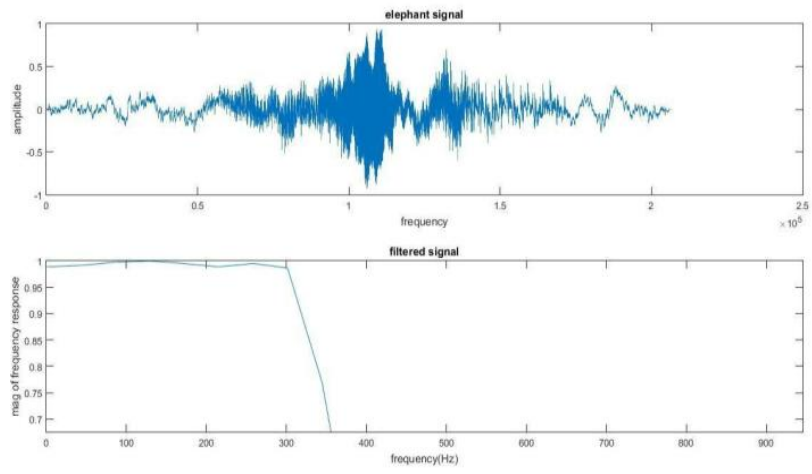

Fig. 12: (a) Elephant signal (b) output of an Elliptic Filter

\section{b) Noise removal}

Practically, the signal is obtained with noise. Noise is unwanted sound judged to be unpleasant, loud or disruptive to hearing. Analyzing a signal without removal of noise gives the incorrect results. So, the noise is removed by applying a filter to the signal. After applying this, the output is a filtered signal which is noisefree. Any method for the removal of noise cannot remove the noise completely, instead it will reduce the noise. Comparison of Fig. 13 (a) and Fig.13 (c) signal, clearly says that there is a reduction of noise. It cannot remove the noise completely but it will reduce the noise compared to the initial signal. Fig.13 (b) shows the Elliptic Filter output.
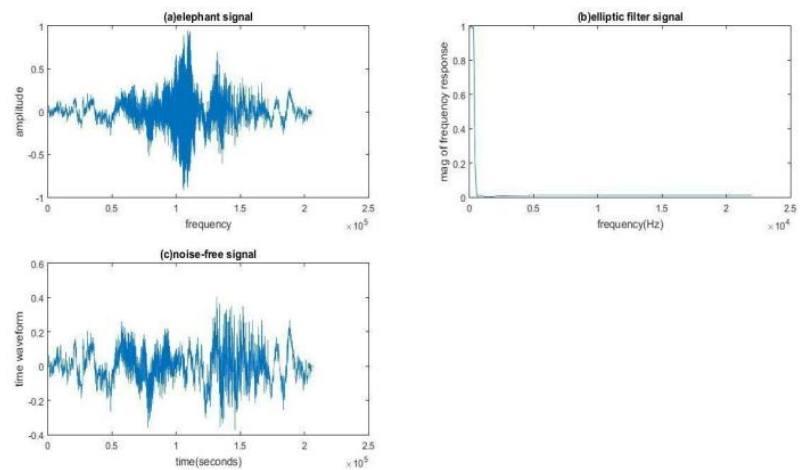

Fig. 13: (a) Elephant Signal (b) Output of an Elliptic Filter (c) Noise Free Signal 


\section{c) Fast Fourier Transform (FFT)}

After removing noise from the signal, FFT is applied to the signal in comparison to the input. The output of FFT contains magnitude response and phase response as plotted as shown in Fig. 14.
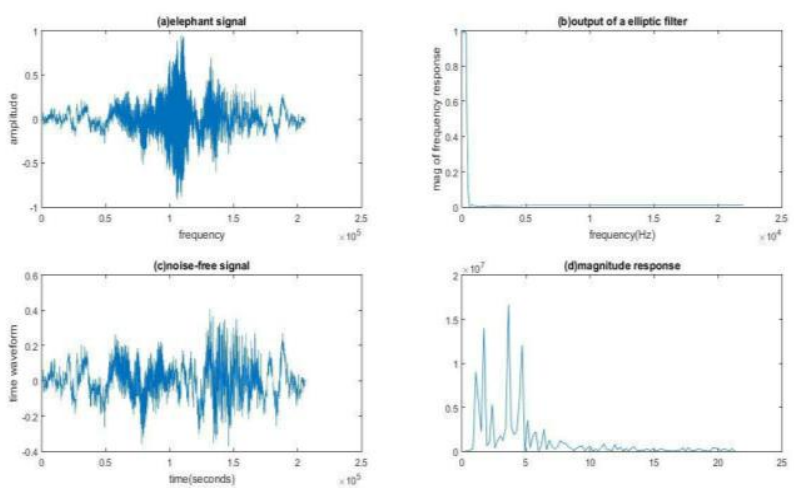

Fig. 14: (a) Elephant Signal (b) Output of an Elliptic Filter (c) Noise-free signal (d) Magnitude Response of the Signal

\section{d) Peak analysis}

Finding peaks in a signal will help us to find the area where the maximum frequencies are present.
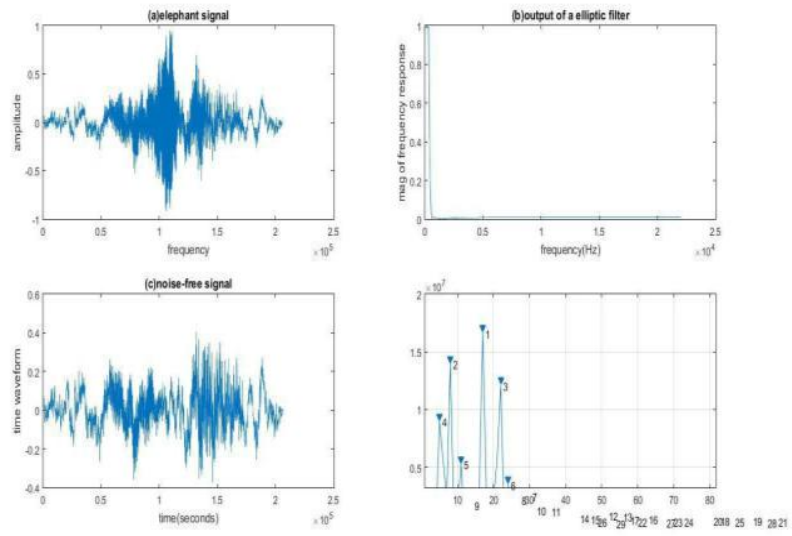

Fig. 15: (a) Elephant signal (b) Output of an Elliptic Filter (c) Noise Free Signal (d) Magnitude response of the Signal with Peaks

By writing the code for peak analysis in MATLAB, the peaks of the signal are determined. In MATLAB, directly the first three peak values of a signal and the power related to that frequencies can be obtained. The peaks in the signal are displayed as in Fig. 15(d).

Peak values displayed in command window is shown below in Fig. 16.
The different types of elephant signals are collected and above analysis is performed for all the different signals using the same procedure described above. The values are tabulated as follows.

Table 1: Frequencies of Different Elephant Signals

\begin{tabular}{|c|c|c|c|c|c|c|}
\hline Signal & F1 & P1 & F2 & P2 & F3 & P3 \\
\hline $\begin{array}{l}\text { Female- } \\
\text { Rumble }\end{array}$ & 17.3108 & 2.2846 & 1.2492 & 1.3935 & 16.9539 & 1.3424 \\
\hline $\begin{array}{c}\text { Male- } \\
\text { Rumble }\end{array}$ & 12.2882 & 2.6585 & 11.5359 & 2.2186 & 10.7836 & 2.1039 \\
\hline $\begin{array}{c}\text { Calf- } \\
\text { Rumble } \\
\text { Rev } \\
\text { Rumble- } \\
\text { Roar }\end{array}$ & $\begin{array}{l}2.4755 \\
4.0238 \\
3.6247\end{array}$ & $\begin{array}{l}1.4697 \\
1.3002 \\
1.6639\end{array}$ & $\begin{array}{l}2.8291 \\
127.4218 \\
1.7057\end{array}$ & $\begin{array}{l}1.0923 \\
0.6451 \\
1.3951\end{array}$ & $\begin{array}{l}3.1827 \\
84.5007 \\
4.6908\end{array}$ & $\begin{array}{l}1.0243 \\
0.1136 \\
1.2093\end{array}$ \\
\hline $\begin{array}{l}\text { Chirping } \\
\text { Trumpet }\end{array}$ & $\begin{array}{l}17.0986 \\
0.9421\end{array}$ & $\begin{array}{l}1.1923 \\
5.0751\end{array}$ & $\begin{array}{r}9.1458 \\
2.5907\end{array}$ & $\begin{array}{l}0.8029 \\
2.6106\end{array}$ & $\begin{array}{l}13.7187 \\
6.1235\end{array}$ & $\begin{array}{l}0.7063 \\
0.9033\end{array}$ \\
\hline $\begin{array}{c}\text { Croaking } \\
\text { by Gail }\end{array}$ & 2.0019 & 4.9 & 2.4189 & 3.6886 & 1.418 & 2.8501 \\
\hline Malaika & 2.3325 & 3.2646 & 2.1575 & 2.0396 & 1.9826 & 1.7002 \\
\hline $\begin{array}{c}\text { Nasal- } \\
\text { Trumpet }\end{array}$ & 1.6515 & 4.2058 & 3.3029 & 3.9855 & 4.4039 & 2.8795 \\
\hline $\begin{array}{l}\text { Rumble- } \\
\text { cry }\end{array}$ & 4.332 & 1.8208 & 4.0226 & 1.6008 & 13.615 & 0.9462 \\
\hline Snort & 4.0271 & 9.71 & 6.3284 & $5.7051 \mathrm{~s}$ & 5.1777 & 4.3998 \\
\hline $\begin{array}{l}\text { Squelch } \\
\text { Average }\end{array}$ & $\begin{array}{l}1.9879 \\
5.699738\end{array}$ & $\begin{array}{l}7.3438 \\
3.606869\end{array}$ & $\begin{array}{l}0.994 \\
13.51558\end{array}$ & $\begin{array}{l}1.8955 \\
2.2364\end{array}$ & $\begin{array}{l}5.9638 \\
13.27038\end{array}$ & $\begin{array}{l}1.4578 \\
1.664362\end{array}$ \\
\hline
\end{tabular}

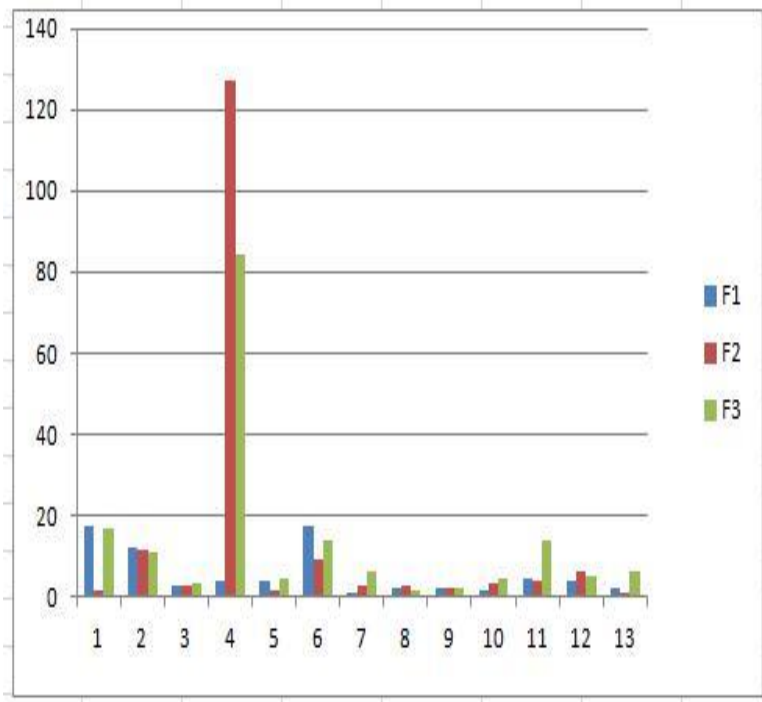

Fig. 17: Frequency Chart

In the Fig. 17, F1, F2, F3 are the first three peak frequencies of different frequencies. From the above chart, it is clear that all the frequencies of elephant sounds lie below $20 \mathrm{~Hz}$ and so that infrasonic detector which is having the range $(0-20 \mathrm{~Hz})$ can be used for detection of elephant signals. As initial stage testing, the output is simulated using MATLAB software. The detected elephant sound is compared with sounds stored in database and output is obtained as shown in Fig. 18 \& Fig. 19. 


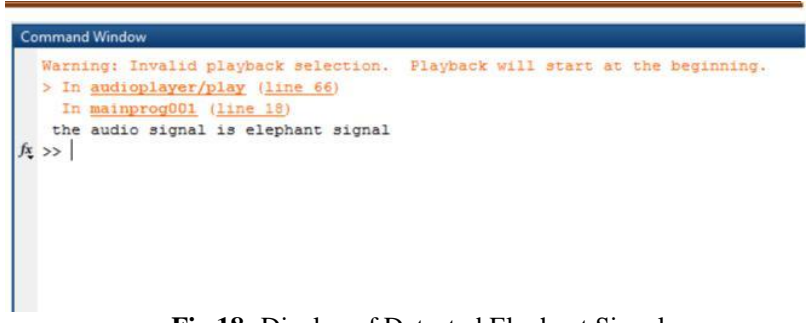

Fig.18: Display of Detected Elephant Signal

If the detected sound is not elephant signal, then honeybee sounds will not be generated and the output displayed is "audio signal is not elephant signal".

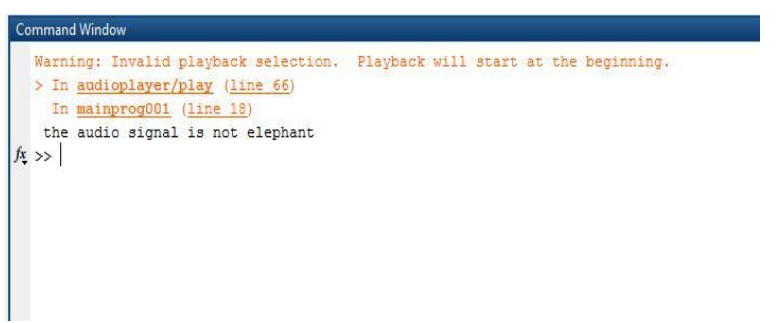

Fig. 19: Display of Non-Elephant Signal

In the Table 2, accuracy of the system can be calculated. To check the accuracy, 20 elephant signal are given as an input to this system and this system can detect 18 signals as elephant signals and generate honey bee sound 2 signals as non-elephant signals and it cannot generate honeybee sounds.

Table 2: Calculation of Accuracy of the system

\begin{tabular}{|c|c|c|c|}
\hline Signal & Number of & True & False \\
\hline $\begin{array}{c}\text { Elephant } \\
\text { signal }\end{array}$ & 20 & 18 & 2 \\
\hline $\begin{array}{c}\text { Non- Elephant } \\
\text { signal }\end{array}$ & 10 & 8 & 2 \\
\hline
\end{tabular}

After this, 10 non-elephant signals (like dog, monkey) [1] are given as input to the system and the system can detect 8 signals as nonelephant signals and it cannot generate honeybee sounds. And it can detect 2 signals as elephant signals and it is generating honeybee sounds. From this, the overall percentage of accuracy is $90 \%$ which can be still increased in future work.

\section{Conclusion}

The above analysis, says that the entire frequencies of an elephant signal lie in the range of below of $20 \mathrm{~Hz}$. Infrasound is sound that is lower in frequency than $20 \mathrm{~Hz}$ or cycles per second. Therefore, conclusion can be made as the elephant signals are considered as infrasound and hence infrasonic detector, Acoustic Sensor, etc., can be used for detection. By using this method, elephant sounds are detected and prevention is done by generating the opposite sounds that is Honey Bee sounds. In Future, this method can be developed to detect different sounds of many wild animals by implementing Hardware Module. For the future purpose this method can be developed as Early Warning systems by integrating with IOT (Internet of Things) which alerts the localized people and consultant authority by making calls or sending messages.

\section{References}

[1] Bardeli.R "Similarity search in animal sound databases". IEEE Trans. on MM, 11(1):68-76, 2009.

[2] Campana.B and Keogh.E "A compression-based distance measure for texture, Statistical Analysis and Data Mining", 3(6):381-398, 2010.

[3] Clemins.P.J, M. B. Trawicki, K. Adi, J. Tao, and M. T. Johnson. "Generalized perceptual features for vocalization analysis across multiple species", Proceedings of the IEEE Int. Conf. on Acoustics, Speech and Signal Proc., volume 1, pages 253-256, 2006.

[4] Dissanayake C.M, R. Kotagiri, M. N. Halgamuge, B. Moran, and P. Farrell "Propagation constraints in elephant localization using an acoustic sensor network" 6th IEEE Conference on Information and Automation for Sustainability, pages 101-105, 2012.

[5] Duda R.O, P. E. Hart, and D. G. Stork. "Pattern classification", Wiley, 2nd edition, 2001.

[6] Greenwood.D. "Critical bandwidth and the frequency coordinates of the basilar membrane", The Journal of the Acoustical Society of America, 33:1344-1356, 1961.

[7] Hao.Y, B. Campana, and E. Keogh "Monitoring and mining animal sounds in visual space", Journal of Insect Behavior, 26(4): 466-493, 2012.

[8] Harris.C and M. Stephens. "A combined corner and edge detector". In 4th Alvey vision conference, pages 147-151. Manchester, UK, 1988.

[9] Langbauer W.R, K. B. Payne, R. A. Charif, L. Rapaport, and F. Osborn. "African elephants respond to distant playbacks of lowfrequency conspecific calls", Journal of Experimental Biology, 157(1):35\{46, 1991.

[10] Mellinger .D.K and C. W. Clark. "Recognizing transient lowfrequency whale sounds by spectrogram correlation", The Journal of the Acoustical Society of America, 107(6):3518-3529, 2000.

[11] Messaoud Z.B, D. Gargouri, S. Zribi, A. Hamida, "Formant Tracking Linear Prediction Model using HMMs for Noisy Speech Processing", International Journal of Signal Processing, Vol. 5 No. 4, pp 291-296,2009

[12] Payne K.B, J. W. R. Langbauer, and E. M. Thomas. "Infrasonic calls of the asian elephant (elephas maximus)". Behavioral Ecology and Sociobiology, 18(4): 297\{301, 1986.

[13] Santiapillai.C, S. Wijeyamohan, G. Bandara, R. Athurupana, N. Dissanayake, and B. Read. "An assessment of the human-elephant conflict in Sri Lanka", Ceylon Journal of Science, 39(1):21-33, 2010.

[14] Seneviratne.L, G. Rossel, H. L. Gunasekera, Y. Madanayake, and G. Doluweera "Elephant infrasound calls as a method for electronic elephant detection", Proceedings of the Symptoms on Human- Elephant Relationships and Conflicts, pages 1-7, 2004.

[15] Stoger.A, G. Heilmann, M. Zeppelzauer, A. Ganswindt, S. Hensman, and B. Charlton. "Visualizing sound emission of elephant vocalizations: Evidence for two rumble production types", PloS one, 7(11): e48907, 2012.

[16] Tracking elephants with GPS collar, Centre for Conservation \&Research,www.ccrsl.org/CCR/Programs/ElephantTracking/Tracki ng GPS.htm

[17] Venter P.J and J. J. Hanekom. "Automatic detection of african elephant (loxodonta africana) infrasonic vocalisations from recordings" Biosystems engineering, 106(3):286-294, 2010.

[18] Weisstein, Eric W."CRC Concise Encyclopedia of Mathematics. CRC Press, ISBN 1-58488- 347-2. (2003).

[19] Wijayakulasooriya.J.Z "Automatic recognition of elephant infrasound calls using formant analysis and hidden markov model", 6th IEEE Int. Conf. on Industrial and Information Sys., pages 244$248,2011$.

[20] Wood.J.D, B. McCowan, W. Langbauer, J. Viljoen, and L. Hart. "Classification of african elephant loxodonta africana rumbles using acoustic parameters and cluster analysis". Bioacoustics, 15(2):143161,2005

[21] Zeppelzauer M, Stoeger AS, Breiteneder C. "Acoustic detection of elephant presence in noisy environments". In: Proceedings of the 2nd ACM International Workshop on Multimedia Analysis for Ecological Data, New York, October 22, Barcelona, Spain ACM Press; p. 3-8, 2013.

[22] Zeppelzauer M, Hensman S, Stoeger S. "Towards an automated acoustic detection system for free-ranging elephants", Bioacoustics 24:13-29, 2015.

[23] Zeppelzauer M, Stoeger S. "Establishing the fundamentals for an elephant early warning and monitoring system", BMC Res Notes $8: 409,2015$. 